\title{
A SEQUENCE OF INTEGERS RELATED TO THE BESSEL FUNCTIONS
}

\author{
L. CARLITZ ${ }^{1}$
}

Let $j_{v, r}$ denote the zeros of $z^{-r} J_{v}(z)$, where $J_{v}(z)$ is the Bessel function of the first kind, and put

$$
\sigma_{2 n}(\nu)=\sum_{r=1}^{\infty}\left(j_{v, r}\right)^{-2 n} \quad(n=1,2,3, \cdots) .
$$

Properties of $\sigma_{2 n}(\nu)$ have been discussed in a recent paper by Kishore [2]. We remark that $\sigma_{2 n}(\nu)$ is a rational function of $\nu$ with rational coefficients; the first twelve functions have been computed by Lehmer [3].

For $\nu= \pm \frac{1}{2}, \sigma_{2 n}(\nu)$ is expressible in terms of the numbers of Bernoulli and Genocchi by means of the following formulas:

$$
\begin{aligned}
\sigma_{2 n}\left(\frac{1}{2}\right) & =(-1)^{n-1} \frac{2^{2 n-1}}{(2 n) !} B_{2 n}, \\
\sigma_{2 n}\left(-\frac{1}{2}\right) & =(-1)^{n} \frac{2^{2 n-2}}{(2 n) !} G_{2 n},
\end{aligned}
$$

where

$$
B^{n}=(B+1)^{n} \quad(n \neq 1), \quad G_{n}=2\left(1-2^{n}\right) B_{n} .
$$

In view of the known arithmetic properties of these and related numbers, it is of some interest to look for arithmetic properties of $\sigma_{2 n}(\nu)$ for other values of $\nu$. In the present note we consider the case $\nu=0$. Elsewhere [1] the writer has discussed the coefficients of $\left(J_{0}(\nu)\right)^{-1}$.

It will be convenient to define

$$
a_{r}=2^{2 r} r !(r-1) ! \sigma_{2 r}(0) \quad(r \geqq 1) .
$$

Thus the formulas $[2,(14),(22)]$

$$
\begin{aligned}
\sum_{r=1}^{n}(-1)^{r} 2 r(r !)^{2}\left(\begin{array}{c}
n \\
r
\end{array}\right)\left(\begin{array}{c}
\nu+n \\
r
\end{array}\right) \sigma_{2 r}(\nu)+n & =0, \\
(\nu+n) \sigma_{2 n}(\nu) & =\sum_{r=1}^{n-1} \sigma_{2 r}(\nu) \sigma_{2 n-2 r}(\nu)
\end{aligned}
$$

reduce to

Received by the editors February 1, 1962.

${ }^{1}$ Supported in part by National Science Foundation grants G16485, G14636. 


$$
\begin{array}{ll}
\sum_{r=1}^{n}(-1)^{r}\left(\begin{array}{l}
n \\
r
\end{array}\right)\left(\begin{array}{l}
n-1 \\
r-1
\end{array}\right) a_{r}+1=0 & (n \geqq 1), \\
a_{n+1}=\sum_{r=1}^{n}\left(\begin{array}{l}
n \\
r
\end{array}\right)\left(\begin{array}{c}
n \\
r-1
\end{array}\right) a_{r} a_{n-r+1} & (n \geqq 1),
\end{array}
$$

respectively.

Since $a_{1}=1$ we find, using either (3) or (4), that

$$
\begin{aligned}
& a_{2}=1, \\
& a_{3}=2^{2}, \\
& a_{4}=3 \cdot 11, \\
& a_{5}=2^{3} \cdot 3 \cdot 19, \\
& a_{6}=2^{2} \cdot 5 \cdot 11 \cdot 43, \\
& a_{7}=2^{4} \cdot 3 \cdot 5^{2} \cdot 229, \\
& a_{8}=3 \cdot 5 \cdot 7 \cdot 167 \cdot 607 .
\end{aligned}
$$

It is evident from (4) that the $a_{n}$ are positive integers. If $n=p$, a prime, it follows from (3) that

$$
a_{p} \equiv 1(\bmod p)
$$

This result can be extended. We recall that if [4]

$$
\begin{aligned}
& n=n_{0}+n_{1} p+n_{2} p^{2}+\cdots \\
& \left(0 \leqq n_{j}<p\right), \\
& r=r_{0}+r_{1} p+r_{2} p^{2}+\cdots \\
& \left(0 \leqq r_{j}<p\right)
\end{aligned}
$$

then

(6)

$$
\left(\begin{array}{l}
n \\
r
\end{array}\right) \equiv\left(\begin{array}{l}
n_{0} \\
r_{0}
\end{array}\right)\left(\begin{array}{l}
n_{1} \\
r_{1}
\end{array}\right)\left(\begin{array}{l}
n_{2} \\
r_{2}
\end{array}\right) \cdots(\bmod p) .
$$

In particular (6) implies

$$
\left(\begin{array}{c}
m p \\
r p
\end{array}\right) \equiv\left(\begin{array}{c}
m \\
r
\end{array}\right), \quad\left(\begin{array}{c}
m p-1 \\
r p-1
\end{array}\right) \equiv\left(\begin{array}{c}
m-1 \\
r-1
\end{array}\right)(\bmod p)
$$

and

$$
\left(\begin{array}{c}
m p \\
r
\end{array}\right) \equiv 0(\bmod p)
$$

Thus if $n=m p$, (3) becomes

$$
\sum_{r=1}^{m}(-1)^{r}\left(\begin{array}{c}
m \\
r
\end{array}\right)\left(\begin{array}{c}
m-1 \\
r-1
\end{array}\right) a_{r p}+1 \equiv 0(\bmod p) .
$$


Comparing (7) with (3) it is evident that

$$
a_{m p} \equiv a_{m}(\bmod p)
$$

and somewhat more generally

$$
a_{m p^{r}} \equiv a_{m}(\bmod p) \quad(r=1,2,3, \cdots) .
$$

We show next that

$$
a_{n} \equiv 0(\bmod p)
$$$$
(p<n<2 p) .
$$

For $n=p+1$, this is obvious from (4). Assuming that (10) holds up to and including the value $n,(4)$ implies

$$
a_{n+1} \equiv \sum_{r=n-p+1}^{p}\left(\begin{array}{l}
n \\
r
\end{array}\right)\left(\begin{array}{c}
n \\
r-1
\end{array}\right) a_{r} a_{n-r+1}(\bmod p) \text {. }
$$

If $n+1=p+m$, where $1 \leqq m<p$, then by (6)

$$
\begin{aligned}
\left(\begin{array}{c}
n \\
r
\end{array}\right) & =\left(\begin{array}{c}
p+m-1 \\
r
\end{array}\right) \equiv\left(\begin{array}{c}
m-1 \\
r
\end{array}\right) \equiv 0(\bmod p) \quad(m \leqq r<p), \\
\left(\begin{array}{c}
n \\
p-1
\end{array}\right) & =\left(\begin{array}{c}
p+m-1 \\
p-1
\end{array}\right) \equiv\left(\begin{array}{c}
m-1 \\
p-1
\end{array}\right) \equiv 0(\bmod p) .
\end{aligned}
$$

Thus $(11)$ reduces to $a_{n+1} \equiv 0(\bmod p)$. This completes the proof of (10).

It is now easy to prove the more general congruence

$$
a_{n} \equiv 0(\bmod p) \quad(n>p, p \nmid n)
$$

by induction. Indeed if

$$
n+1=k p+m, \quad 1 \leqq m<p,
$$

then by (6) and the inductive hypothesis

$$
a_{n+1} \equiv 2\left(\begin{array}{c}
n \\
m
\end{array}\right)\left(\begin{array}{c}
n \\
m-1
\end{array}\right) a_{m} a_{k p}(\bmod p) ;
$$

but by (6)

$$
\left(\begin{array}{l}
n \\
m
\end{array}\right)=\left(\begin{array}{c}
k p+m-1 \\
m
\end{array}\right) \equiv\left(\begin{array}{c}
m-1 \\
m
\end{array}\right) \equiv 0,
$$

so that $a_{n+1} \equiv 0(\bmod p)$.

We remark that

$$
a_{n} \equiv 0(\bmod n-1) \quad(n>1)
$$


Indeed if we put

$$
a_{n}=(n-1) b_{n}
$$

(4) becomes for $n>1$

$$
\begin{aligned}
n b_{n+1} & =2 n(n-1) b_{n}+\sum_{r=2}^{n-1}\left(\begin{array}{c}
n \\
r
\end{array}\right)\left(\begin{array}{c}
n \\
r-1
\end{array}\right)(n-r)(r-1) b_{r} b_{n-r+1} \\
& =2 n(n-1) b_{n}+n^{2} \sum_{r=2}^{n-1}\left(\begin{array}{c}
n-1 \\
r
\end{array}\right)\left(\begin{array}{c}
n-1 \\
r-2
\end{array}\right) b_{r} b_{n-r+1},
\end{aligned}
$$

so that

$$
b_{n+1}=2(n-1) b_{n}+n \sum_{r=2}^{n-1}\left(\begin{array}{c}
n-1 \\
r
\end{array}\right)\left(\begin{array}{c}
n-1 \\
r-2
\end{array}\right) b_{r} b_{n-r+1} \quad(n>1) .
$$

Since $b_{2}=1$ it is evident from (14) that $b_{n}$ is integral for all $n \geqq 2$. This proves (13).

Returning to (4) it is clear that

$$
a_{p+1} \equiv 2 p a_{p}\left(\bmod p^{2}\right)
$$

Combining this with (5) we get

$$
a_{p+1} \equiv 2 p\left(\bmod p^{2}\right) .
$$

Similarly we have for $p>2$

$$
a_{p+2} \equiv 2 a_{p+1}+2\left(\begin{array}{c}
p+1 \\
2
\end{array}\right)(p+1) a_{p}\left(\bmod p^{2}\right) .
$$

Using (5) and (15) this reduces to

$$
a_{p+2} \equiv 5 p\left(\bmod p^{2}\right) .
$$

In the same way if $p>3$ we get

$$
\begin{aligned}
a_{p+3} \equiv & 2 a_{p+2}+2 \cdot\left(\begin{array}{c}
p+2 \\
2
\end{array}\right)(p+2) a_{p+1} \\
& +8\left(\begin{array}{c}
p+2 \\
3
\end{array}\right)\left(\begin{array}{c}
p+2 \\
2
\end{array}\right) a_{p}\left(\bmod p^{2}\right)
\end{aligned}
$$

which reduces to

$$
a_{p+3} \equiv \frac{92}{3} p\left(\bmod p^{2}\right) \quad(p>3)
$$

To get a general result of this kind we put 


$$
a_{p+n} \equiv p c_{n}^{(p)}\left(\bmod p^{2}\right) \quad(0<n<p),
$$

so that by (10) $c_{n}^{(p)}$ is integral. Now by (4)

$$
\begin{aligned}
a_{p+n} & =\sum_{r=1}^{p+n-1}\left(\begin{array}{c}
p+n-1 \\
r
\end{array}\right)\left(\begin{array}{c}
p+n-1 \\
r-1
\end{array}\right) a_{r} a_{p+n-r} \\
& \equiv 2 \sum_{r=1}^{n}\left(\begin{array}{c}
p+n-1 \\
r
\end{array}\right)\left(\begin{array}{c}
p+n-1 \\
r-1
\end{array}\right) a_{r} a_{p+n-r}\left(\bmod p^{2}\right) .
\end{aligned}
$$

Making use of (18) and (6) this becomes

(19) $c_{n}^{(p)} \equiv 2 \sum_{r=1}^{n-1}\left(\begin{array}{c}n-1 \\ r\end{array}\right)\left(\begin{array}{c}n-1 \\ r-1\end{array}\right) a_{r} c_{n-r}^{(p)}+\frac{2}{n} a_{n}(\bmod p)(1<n<p)$.

By means of (19) the $c_{n}^{(p)}$ can be computed. However, it is simpler to define a single sequence $\left\{c_{n}\right\}$ by means of

$$
c_{n}=2 \sum_{r=1}^{n-1}\left(\begin{array}{c}
n-1 \\
r
\end{array}\right)\left(\begin{array}{c}
n-1 \\
r-1
\end{array}\right) a_{r} c_{n \rightarrow r}+\frac{2}{n} a_{n} \quad(n>1)
$$

with $c_{1}=2$. We evidently have

$$
c_{n}^{(p)} \equiv c_{n}(\bmod p) \quad(0<n<p) .
$$

The $c_{n}$ as defined by (20) are not integral. If we put

$$
c_{n}^{\prime}=n c_{n} \quad(n \geqq 1), \quad c_{0}=1,
$$

then (20) becomes

$$
c_{n}^{\prime}=2 \sum_{r=1}^{n}\left(\begin{array}{l}
n \\
r
\end{array}\right)\left(\begin{array}{l}
n-1 \\
r-1
\end{array}\right) a_{r}^{\prime} c_{n-r} \quad(n \geqq 1) ;
$$

the $c_{n}^{\prime}$ are therefore integral. Moreover it follows from (23) that

$$
\sum_{n=1}^{\infty} \frac{c_{n}^{\prime}\left(\frac{x}{2}\right)^{2 n}}{n !(n-1) !}=2 \sum_{r=1}^{\infty} \frac{a_{r}\left(\frac{x}{2}\right)^{2 n}}{r !(r-1) !} \sum_{n=0}^{\infty} \frac{c_{n}^{\prime}\left(\frac{x}{2}\right)^{2 n}}{n ! n !} .
$$

If we put

$$
C(x)=\sum_{n=0}^{\infty} \frac{c_{n}^{\prime}\left(\frac{x}{2}\right)^{2 n}}{n ! n !}, \quad A(x)=\sum_{r=1}^{\infty} \frac{a_{r}\left(\frac{x}{2}\right)^{2 n}}{r !(r-1) !}
$$

then 


$$
\frac{x}{2} C^{\prime}(x)=\sum_{n=1}^{\infty} \frac{c_{n}^{\prime}\left(\frac{x}{2}\right)^{2 n}}{n !(n-1) !}
$$

so that

$$
\frac{x}{2} C^{\prime}(x)=2 A(x) C(x)
$$

But [2]

$$
A(x)=\sum_{r=1}^{\infty} \sigma_{2 r}(0) x^{2 r}=-\frac{1}{2} x \frac{J_{0}^{\prime}(x)}{J_{0}(x)} .
$$

It follows that

$$
\frac{C^{\prime}(x)}{C(x)}=-\frac{J_{0}^{\prime}(x)}{J_{0}(x)}
$$

which yields

$$
C(x)=\left(J_{0}(x)\right)^{-2} .
$$

We have accordingly found a simple generating function for the $c_{n}$. It follows from $(13)$ that if $n \equiv 1\left(\bmod p^{k}\right)$ then $a_{n} \equiv 1\left(\bmod p^{k}\right)$. Using (4) and (8) it is not difficult to show that if $n=m p^{k}+1$ then

$$
a_{n} \equiv m p^{k} a_{m}\left(\bmod p^{k+1}\right) .
$$

We shall now show that if

$$
n=n_{s} p^{s}+n_{s+1} p^{s+1}+\cdots+n_{t} p^{2} \quad\left(0 \leqq n_{j}<p\right)
$$

and $n_{s} \geqq 1, n_{t} \geqq 1$, then

$$
a_{n} \equiv 0\left(\bmod p^{t-s}\right) \text {. }
$$

The proof is by induction on $n$. We use (4) with $n$ replaced by $n-1$. Let $1 \leqq r<n$ and put

$$
\begin{aligned}
& r=r_{\ell^{\prime}} p^{*^{\prime}}+r_{t^{\prime}+1} p^{\alpha^{\prime}+1}+\cdots+r_{t^{\prime}} p^{t^{\prime}} \quad\left(0 \leqq r_{j}<p\right),
\end{aligned}
$$

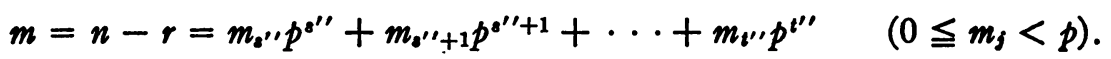

Clearly either $s^{\prime} \leqq s$ or $s^{\prime \prime} \leqq s$; also $t^{\prime} \leqq t$ and $t^{\prime \prime} \leqq t$. If $t^{\prime}=t^{\prime \prime}=t$ there is evidently nothing to prove.

(i) $t^{\prime}=t, t^{\prime \prime}<t$. By the inductive hypothesis

$$
a_{r} \equiv 0\left(\bmod p^{t^{\prime}-s^{\prime}}\right), \quad a_{n \rightarrow r} \equiv 0\left(\bmod p^{t^{\prime \prime}-s^{\prime \prime}}\right)
$$


so that

$$
a_{r} a_{n \rightarrow r} \equiv 0\left(\bmod p^{t^{\prime}+t^{\prime \prime}-\mathbf{s}^{\prime}-\mathbf{s}^{\prime \prime}}\right) .
$$

If $s^{\prime} \leqq t^{\prime \prime}$ it follows (since $t^{\prime \prime} \geqq \max \left(s^{\prime}, s^{\prime \prime}\right)$ ) that $t^{\prime}+t^{\prime \prime}-s^{\prime}-s^{\prime \prime} \geqq t-s$. If however $s^{\prime}>t^{\prime \prime}$ we examine the binomial coefficient $C_{n-1, n}$.

We recall that if

$$
\begin{array}{rlrl}
n & =n_{0}+n_{1} p+\cdots+n_{k} p^{k} \quad\left(0 \leqq n_{j}<p\right), \\
S(n) & =n_{0}+n_{1}+\cdots+n_{k}, &
\end{array}
$$

then $n$ ! is divisible by exactly $p^{e}$, where

$$
(p-1)^{\circ}=n-S(n) \text {. }
$$

It follows that $\left(\begin{array}{c}n-1 \\ r\end{array}\right)$ is divisible by $p^{\mathbf{s}^{\prime}-\mathrm{s}^{\prime \prime}}$. Since $\left(t^{\prime}+t^{\prime \prime}-s^{\prime}-s^{\prime \prime}\right)$ $+\left(s^{\prime}-s^{\prime \prime}\right)=t+t^{\prime \prime}-2 s \geqq t-s$ we get

$$
\left(\begin{array}{c}
n-1 \\
r
\end{array}\right) a_{r} a_{n-r} \equiv 0\left(\bmod p^{t-s}\right) \text {. }
$$

(ii) $t^{\prime}<t, t^{\prime \prime}<t$. We may suppose that $t^{\prime \prime} \leqq t^{\prime}=t-1$. Also it is clear that $s^{\prime} \leqq t^{\prime \prime}$. Then

$$
t^{\prime}+t^{\prime \prime}-s^{\prime}-s^{\prime \prime} \geqq t-s-1 ;
$$

indeed if $s \min \left(s^{\prime}, s^{\prime \prime}\right)$ we get

$$
t^{\prime}+t^{\prime \prime}-s^{\prime}-s^{\prime \prime} \geqq t-s .
$$

Thus only the case $s=\min \left(s^{\prime}, s^{\prime \prime}\right)$ requires further examination. With the present hypothesis we evidently have

$$
\left(\begin{array}{c}
n \\
r
\end{array}\right) \equiv 0(\bmod p) \text {; }
$$

but when $s=\min \left(s^{\prime}, s^{\prime \prime}\right)$ then either $n$ and $r$ or $n$ and $n-r$ are divisible by the same power of $p$. It follows that either

$$
\left(\begin{array}{c}
n-1 \\
r
\end{array}\right) \text { or }\left(\begin{array}{c}
n-1 \\
r-1
\end{array}\right)
$$

is divisible by $p$. Consequently

$$
\left(\begin{array}{c}
n-1 \\
r
\end{array}\right)\left(\begin{array}{c}
n-1 \\
r-1
\end{array}\right) a_{r} a_{n \rightarrow r} \equiv 0\left(\bmod p^{t-s}\right)
$$

This completes the proof of (26).

SUMmaRY. The sequence of positive integers $\left\{a_{n}\right\}$ defined by (2) -or alternatively by (3) or (4) - have the following properties. 
1. $a_{m p} \equiv a_{m}, \quad a_{p} \equiv 1(\bmod p)$.

2. $a_{n} \equiv 0(\bmod p)$

$$
\begin{array}{r}
(n>p, p+n) . \\
(n>1) . \\
(1 \leqq n<p),
\end{array}
$$

4. $a_{p+n} \equiv c_{n} p\left(\bmod p^{2}\right)$

where the $c_{n}$ are defined by

$$
1+\sum_{n=1}^{\infty} \frac{c_{n}\left(\frac{x}{2}\right)^{2 n}}{(n-1) !(n-1) !}=\left(J_{0}(x)\right)^{-2} ;
$$

moreover $n c_{n}$ is integral.

5. If $n=m p^{k}+1$ then

$$
a_{n} \equiv m p^{k} a_{m}\left(\bmod p^{k+1}\right) .
$$

6. If $p^{s} \mid n, p^{2+1} \nmid n, p^{t} \leqq n<p^{t+1}$ then

$$
a_{n} \equiv 0\left(\bmod p^{t-s}\right) \text {. }
$$

The following values of $a_{n}$ were computed by $\mathrm{R}$. Carlitz in the Duke University Computing Laboratory.

$$
\begin{aligned}
& a_{9}=2^{4} \cdot 5 \cdot 7 \cdot 19 \cdot 31 \cdot 1607 \\
& a_{10}=2^{3} \cdot 3^{2} \cdot 7 \cdot 199 \cdot 328981 \\
& a_{11}=2^{5} \cdot 3^{2} \cdot 5 \cdot 7 \cdot 83 \cdot 3000553 \\
& a_{12}=2^{2} \cdot 3 \cdot 5^{2} \cdot 7 \cdot 11 \cdot 2389 \cdot 4153159 \\
& a_{13}=2^{5} \cdot 3^{2} \cdot 5 \cdot 74 \cdot 11 \cdot 29 \cdot 97 \cdot 139 \cdot 1663 \\
& a_{14}=2^{4} \cdot 3^{2} \cdot 5 \cdot 11 \cdot 13 \cdot 37 \cdot 107 \cdot 1283 \cdot 5952613 \\
& a_{16}=2^{6} \cdot 3 \cdot 7 \cdot 11 \cdot 13 \cdot 23 \cdot 563 \cdot 797 \cdot 227966279 \\
& a_{16}=3^{2} \cdot 5 \cdot 7 \cdot 11 \cdot 13 \cdot 17 \cdot 41 \cdot 2390700514417253 \\
& a_{17}=2^{5} \cdot 3^{2} \cdot 5^{3} \cdot 7 \cdot 11 \cdot 13 \cdot 19 \cdot 461 \cdot 1342361 \cdot 33327739 \\
& a_{18}=2^{4} \cdot 5 \cdot 7 \cdot 11 \cdot 13 \cdot 17 \cdot 79 \cdot 199729 \cdot 139135943558279 \\
& a_{19}=2^{6} \cdot 3^{2} \cdot 5 \cdot 7 \cdot 11 \cdot 13^{2} \cdot 17 \cdot a_{19}^{\prime} \\
& a_{20}=2^{3} \cdot 3^{2} \cdot 7^{2} \cdot 11 \cdot 13 \cdot 17 \cdot 19 \cdot 1109 \cdot a_{20}^{\prime} \\
& a_{21}=2^{6} \cdot 3 \cdot 5 \cdot 11 \cdot 13 \cdot 17 \cdot 19 \cdot 47 \cdot a_{21}^{\prime} \\
& a_{22}=2^{5} \cdot 3^{2} \cdot 5^{2} \cdot 7 \cdot 13 \cdot 17 \cdot 19 \cdot a_{22}^{\prime} \\
& a_{28}=2^{7} \cdot 3^{2} \cdot 5 \cdot 7 \cdot 11 \cdot 13^{2} \cdot 17 \cdot 19 \cdot a_{23}^{\prime} \\
& a_{24}=2^{2} \cdot 3 \cdot 5 \cdot 7 \cdot 11 \cdot 13 \cdot 17 \cdot 19 \cdot 23 \cdot a_{24}^{\prime}
\end{aligned}
$$




$$
\begin{aligned}
& a_{25}=2^{6} \cdot 3^{2} \cdot 7 \cdot 11 \cdot 13 \cdot 17 \cdot 19 \cdot 23 \cdot a_{25}^{\prime} \\
& a_{26}=2^{5} \cdot 3^{2} \cdot 5^{2} \cdot 7 \cdot 11 \cdot 17 \cdot 19 \cdot 23^{2} \cdot 79 \cdot a_{26}^{\prime} \\
& a_{27}=2^{7} \cdot 5^{3} \cdot 7^{2} \cdot 11 \cdot 13 \cdot 17 \cdot 19 \cdot 23 \cdot 29 \cdot 43^{3} \cdot a_{27}^{\prime} \\
& a_{28}=2^{4} \cdot 3^{3} \cdot 5^{2} \cdot 11 \cdot 13 \cdot 17 \cdot 19 \cdot 23 \cdot a_{28}^{\prime} \\
& a_{29}=2^{7} \cdot 3^{3} \cdot 5^{2} \cdot 7 \cdot 11 \cdot 13 \cdot 17 \cdot 19 \cdot 23 \cdot a_{29}^{\prime} \\
& a_{30}=2^{6} \cdot 3^{2} \cdot 5 \cdot 7 \cdot 11 \cdot 13 \cdot 17 \cdot 19 \cdot 23 \cdot 29 \cdot a_{30}^{\prime}
\end{aligned}
$$

The numbers $a_{19}^{\prime}, \cdots, a_{30}^{\prime}$ have not been factored completely but at any rate have no prime divisors $<10^{4}$. The number $a_{30}^{\prime}$ has 47 digits.

\section{REFERENCES}

1. L. Carlitz, The coefficients of the reciprocal of $J_{0}(x)$, Arc. Math. 6 (1955), 121127.

2. N. Kishore, The Rayleigh function, Proc. Amer. Math. Soc. (to appear).

3. D. H. Lehmer, Zeros of the Bessel function $J_{\nu}(x)$, Math. Tables Aids Comput. 1 (1943-45), 405-407.

DUKE UNIVERSITY 